\title{
The LHCb Vertex Detector Upgrade
}

\author{
Marina Artuso*i \\ Syracuse University Syracuse NY 13244 \\ E-mail: artuso@physics.syr.edu
}

The likely discovery of new particles at LHC will be an exciting opportunity to probe physics beyond the Standard Model and will raise new questions that may be elucidated by precision measurements of beauty and charm decays. The LHCb experiment is poised to start this ambitious program when LHC turns on, and is planning to continue it with an upgrade that should enhance its physics sensitivity by at least two orders of magnitude. A new vertex detector is a critical component to achieve this goal. Important requirements are a radiation resistance up to a fluence of about $10^{16} n_{e q} \mathrm{~cm}^{-2}$, and a configuration suitable for use in a fast and efficient vertex trigger. The design concept and technology options considered will be reviewed.

The 16th International Workshop on Vertex detectors

September 23-28 2007

Lake Placid, NY, USA

${ }^{*}$ Speaker.
${ }^{\dagger}$ On behalf of the LHCb VELO group. 


\section{Introduction}

Experimental particle physics is at the eve of a very exciting time that bears to promise of ground breaking discoveries. ATLAS and CMS are poised to explore the mass scale of the order of the electro-weak symmetry breaking, and uncover evidence for exotic new particles that may lead to a better defined path beyond the Standard Model. LHCb, with its precise and systematic exploration of interesting beauty and charm decays, will study how the new particles interfere virtually with $W$ and $Z$ bosons in these decays. These observations can tell us a great deal about the nature of new physics, especially their phases.

The present $\mathrm{LHCb}$ detector has been designed to be able to cope with an instantaneous luminosity up to $\sim 5 \times 10^{32} \mathrm{~cm}^{-2} \mathrm{~s}^{-1}$. This is a factor of 20-50 below the design luminosity of LHC. Thus even without any Super-LHC (SLHC) upgrade, there is ample room to increase sensitivity, provided that the experiment can profit from a higher peak luminosity. The first period of data taking comprises $\sim 10 \mathrm{fb}^{-1}$. The vertex detector (VELO) is the only subsystem that is expected to perform without any significant loss of resolution and efficiency up to an integrated luminosity of about $6 \mathrm{fb}^{-1}$, and thus we are producing replacement modules to be installed during the first data taking stage. In order to achieve the desired sensitivity to be able to uncover new physics effects, even if their manifestation is of very subtle nature, the goal set for the upgraded $\mathrm{LHCb}$ (Super-LHCb) is the accumulation of $\sim 100 \mathrm{fb}^{-1}$ without any detector replacement [1] throughout the duration of this second phase. In addition, we are striving to maximize the sensitivity to many interesting hadronic beauty and charm decay channels, thus we want to implement a heavy flavor trigger algorithm that is very efficient and selective for hadronic final states.

\section{The Luminosity Upgrade}

The bunch crossing rate at the LHCb interaction point (P8) is $40.08 \mathrm{MHz}$, while 2622 out of the theoretically possible 3564 crossings have protons in both bunches. Thus the maximum crossing rate with at least one pp interaction is $\sim 30 \mathrm{MHz}$. Figure 1 shows the change of interaction rates as a function of the luminosity. The top plot shows the number of crossings with at least one visible interaction, while the bottom plot shows the average number of visible interactions per crossing, for events with at least one pp interaction.

Figure 2 shows the impact of the increased occupancy in the trigger yield as a function of luminosity. A channel sensitive to the $\mu$ trigger, $\left(B_{S}^{0} \rightarrow \psi \phi, J / \psi \rightarrow \mu^{+} \mu^{-}\right)$, is compared with a channel sensitive to the general hadron trigger $\left(B s \rightarrow \phi \phi, \phi \rightarrow K^{+} K^{-}\right)$. After the nominal $\mathrm{LHCb}$ luminosity is reached, it is clear that decays relying on the hadron trigger cannot exploit the increased luminosity. Therefore, a key element in the LHCb upgrade is the development of a hadron trigger strategy that maintains its efficiency at higher luminosity. This is particularly important as it ensures that the experiment will be able to uncover new physics in a flexible manner, informed by the knowledge acquired by the other LHC experiments.

A very distinctive feature that can be used in designing hadron triggers that are highly efficient for heavy flavored meson decays produced in the LHCb event environment, while retaining a high degree of rejection of the more common minimum bias events, is the relatively long decay path of charm and beauty mesons. Detached vertex criteria are widely used in experiment operating 


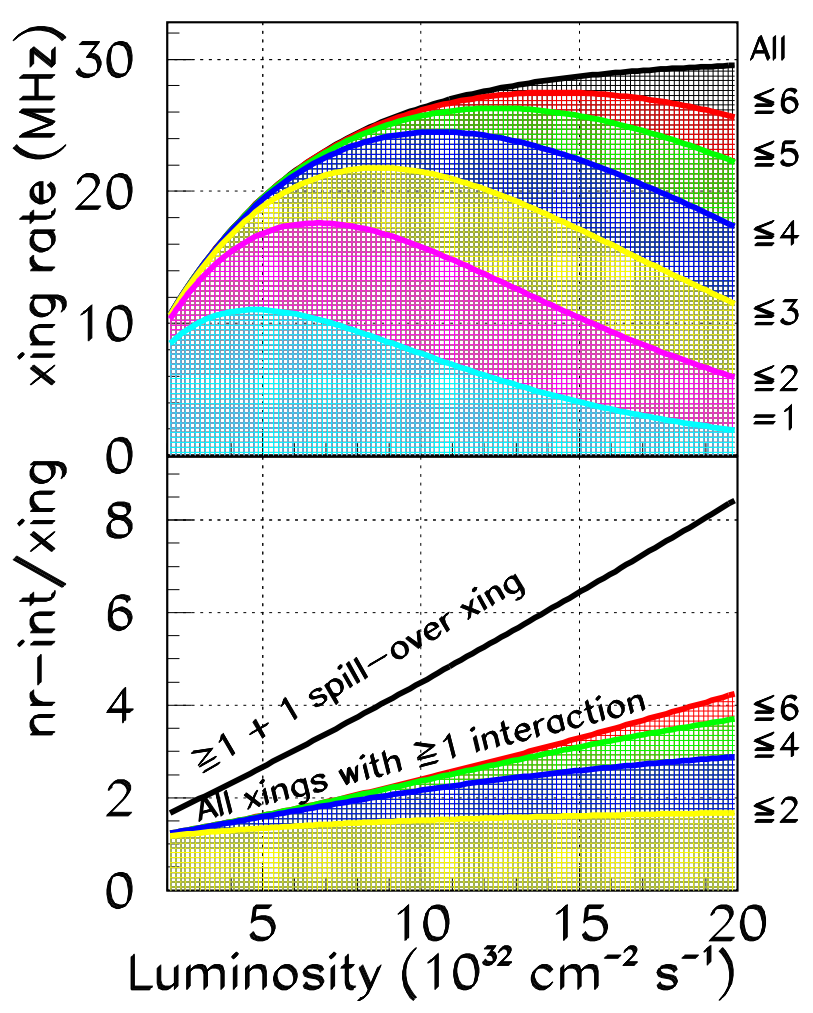

Figure 1: The top plot shows the number of crossings with at least one visible interaction; the bottom plot shows the average number of visible interactions per crossing, for events with at least one pp interaction [2].

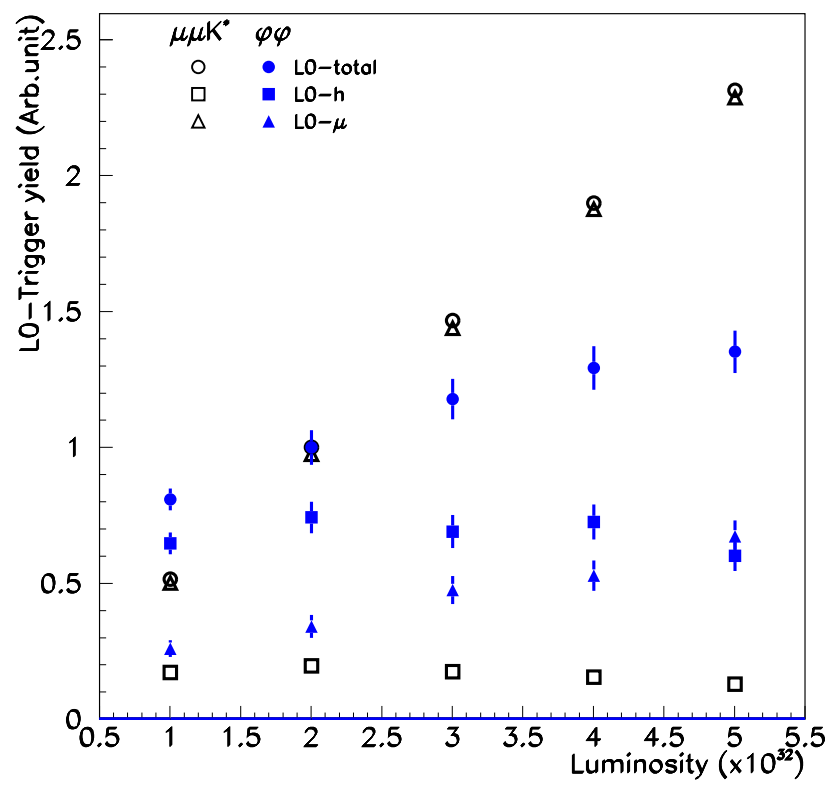

Figure 2: The L0-trigger yield as a function of luminosity for two decay channels: $\mu \mu \mathrm{K}^{*}$ (open points) and $\phi \phi$ (closed points). The total L0-trigger yield, and the contributions from the L0-hadron and muon triggers are shown separately [2]. 


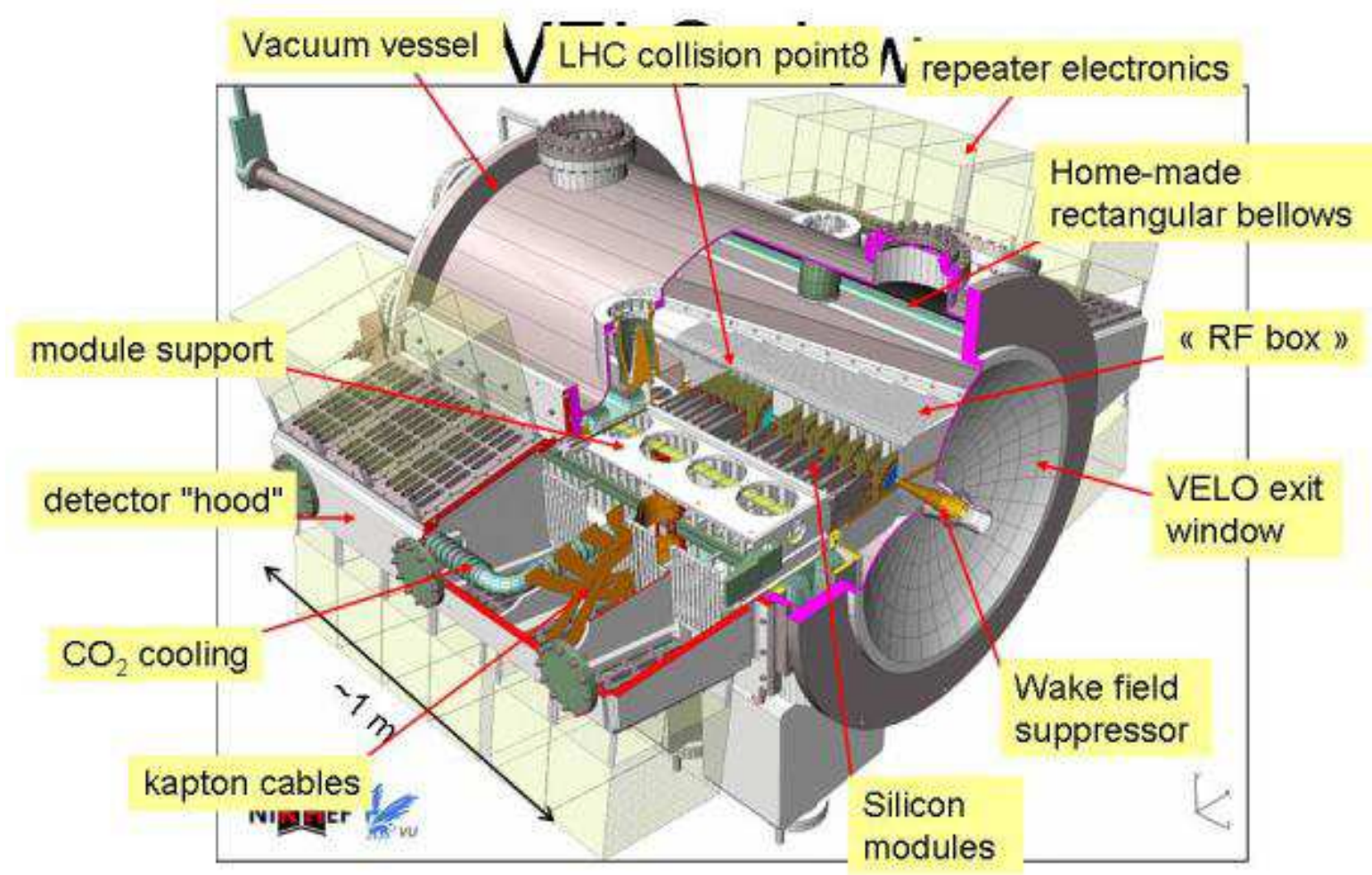

Figure 3: Illustration of the VELO detector layout. The detector is split into tow halves, one of which is shown in this Figure. Each half comprises 21 modules.

at hadron machines, but typically they are introduced in the higher trigger levels. A strategy to incorporate these selection criteria in the lowest trigger level appears to be a promising avenue to implement the more robust and general hadron trigger that is a key component of the $\mathrm{LHCb}$ upgrade. This goal requires a strong connection between the SUPER-LHCb trigger and vertex detector design efforts.

\section{The Present LHCb Vertex Detector System}

The present VErtex LOcator (VELO) silicon detector system consists of 21 stations of silicon strip detectors positioned along the beam and perpendicular to the beam axis, as shown in Figure 3. Each station comprises two double sided modules that cover $1 / 2$ of the acceptance. They are mounted on retractable stages, in order to protect the modules from excessive radiation damage during injection.

Two semi-circular sensors are glued back to back on a hybrid made of a carbon fiber/TPG (thermal pyrolytic graphite) with kapton flex circuits laminated on both sides to form a double 
sided module. The sensor active area span between 8 and $40 \mathrm{~mm}$. Both of them comprise 2048 strips sensitive either to the radial $(\mathrm{R})$ or azimuthal $(\phi)$ coordinate in a cylindrical coordinate system where $\mathrm{Z}$ is along the beam axis. More details can be found in Ref. [3]. The hybrids host 16 front end ASICs (BEETLE) [4] that include 128 low noise and fast analog processors. The analog outputs are stored in a $4 \mu$ s deep analog pipeline and are read out upon the arrival of an L0 trigger signal through 4 LVDS lines, each 32 channel deep.

In addition, there are $4 \mathrm{R}$ sensitive modules that are identified as "VETO stations," which have the purpose of rejecting events with more than 1 interaction. The VETO stations will not be replaced in the planned upgrade, as we plan to process events with multiple interactions efficiently.

The $\mathrm{LHCb}$ detector configuration, which is oriented in the forward direction, implies a highly non-uniform irradiation of the silicon sensors in the vertex telescope. The innermost radius, being so close to the beam, will be exposed to $1.3 \times 10^{14} \mathrm{n}_{\text {eq }} / \mathrm{cm}^{-2}$ per year. Thus the technology chosen for the VELO sensor is the $n^{+}$-on- $n$ [5], adopted by the other LHC experiments for their inner tracking layers, where $n^{+}$strips are implanted on $n$-type substrate. Interstrip isolation is achieved with a blanket p-spray technique [6]. Even with this radiation hard implementation, it is expected that after few years of operation the VELO detector system will need to be replaced. Replacement module production is already underway. The sensor geometry is essentially unchanged, but we have adopted $n^{+}$-on- $p$ fabrication technology, similar to the one used for the present detectors but implemented on p-type substrates. These detectors are easier to fabricate, as they require only single side processing and bear the promise of even greater radiation resistance [7].

\section{SUPER-LHCb Vertex Detector Requirements}

The LHCb vertex detector must enable fast and robust pattern recognition capabilities, as it is going to be the heart of the separation between signal and background events on the basis of the vertex topology. Further improvements on the proper time resolution have a significant impact on the detector performance.

One alternative is a reduction of the amount of material in front of the fist sensing layer in the system. This implies a redesign of the RF shield, which is also the dominant contributor to the average radiation length of this detector system. A drastic solution would be a replacement of the RF shield with wires to screen the beam image currents. This approach may even enable to locate the innermost egde of the modules closer to the beam axis, with additional gains in proper time resolution.

Closely coupled to the goal to achieve small inner radius, and to maintain stable operation during the accumulation of the $100 \mathrm{fb}^{-1}$ data set, is radiation resistance. The innermost region of the detector is expected to receive an integrated fluence of about $10^{16} 1 \mathrm{MeV} \mathrm{n} \mathrm{n}_{\mathrm{eq}} / \mathrm{cm}^{2}$, comparable with the radiation exposure expected for the innermost tracking layers of the central SLHC detectors.

Finally, an overall material minimization for any chosen solution is necessary to optimize the overall performance of the SUPER-LHCb detector system. This implies a careful design of the support and cooling systems, and the careful selection of the number of modules and their composition. 


\section{The EVELO-VESPA Concept}

A first approach to address the requirements described before is the exploration of possible improvements of the existing detector system, to see whether the existing design can be sufficiently enhanced to meet that challenging requirements of SUPER-LHCb. There are three areas to be considered: radiation resistance, readout speed, and trigger performance.

Steps to increase the radiation resistance that are considered are the reduction of the strip area, and improved bias scheme. We need to develop a new front end electronics to push the data out at $40 \mathrm{MHz}$, and relies on a "rate control trigger" largely based on the present HLT trigger algorithm with performance improvements that allow the desired rate suppression. R\&D is needed to validate this approach and to identify the maximum luminosity at which such a strategy is effective. Limitations related to the higher occupancy and more complex pattern recognition as the number of hits increases need to be taken into account.

\section{The PIXEL-VESPA Concept}

An alternative approach relies on hybrid pixel sensors bump bonded to suitable front end electronics. This solution allow the a fast determination of space points that can be aggregated into tracks with relatively simple pattern recognition algorithms. In addition, the use of the pixel geometry would remove the constraint that the system is perfectly mechanically centered around the beam axis, to allow the R-z trigger scheme to operate.

A novel trigger concept can be implemented with this approach, based on the vertex topology. This would be designed to analyze multiple interaction and to select generic beauty and charm decays on the basis of the identification of detached vertices, achieved through precision threedimensional tracking information from the very beginning of the event selection process. Significant advances on this solution were achieved by the BTeV trigger group [8]. The low occupancy, and ease in pattern recognition featured by vertex detector modules implemented with hybrid pixel technology are key elements in this approach.

\subsection{PIXEL-VESPA Technology}

Hybrid pixel devices are our preferred solution because they represent a mature and robust technology that we expect to complete this project in the relatively stringent time scale envisaged ( 2013). This approach is quite mature having been perfected in the extensive BTeV R\&D and in the development and production of the ATLAS and CMS vertex detectors. The hybrid approach allows for independent development and testing of the front end sensors and of the readout electronics.

There are several advantages associated with the reduced pixel area: the low input capacitance seen by the preamplifier in the front end electronics produces a corresponding low intrinsic noise (of the order of $100 \mathrm{e}^{-}$), and a negligible common mode noise. This results in a higher signal to noise ratio than strip devices with the same detector thickness. The extremely low noise occupancy is an advantage for pattern recognition.

In general, sensors with small pixel dimension of approximately $50 \mu \mathrm{m}$ are a well developed technology. Two possible improvements to the standard sensor technology developed for the AT- 
LAS and CMS pixel devices are currently considered. One of them is the implementation pixel devices with the $n^{+}$-on- $p$ approach may present significant advantages in production yields and radiation resistance. A technology that is currently receiving a lot of attention is the so called "3 D” silicon, made using Micro-Electro-Mechanical-Systems (MEMS) processing. In this approach the electrodes penetrate through the silicon bulk perpendicular to the surface. Edge electrodes eliminate the need for guard ring and allow an optimization of the sensor active area. Excellent spatial and time resolution, and radiation resistance have been demonstrated. In addition, as the depletion occurs laterally rather than in the direction of the "depth" of the device, low bias voltages are needed. This feature is very important for highly irradiated devices, as even the operation in partially depleted mode will be extremely challenging in presence of heavy radiation doses. In fact, in order to maintain good charge collection efficiency in standard planar sensors, voltages as high as $1000 \mathrm{~V}$ may be needed [5], while depletion voltages for 3D detectors is of the order of $10 \mathrm{~V}$. This significantly reduces the challenges in cooling and power distribution that are very prominent in the next generation of vertex detectors operating in very adverse radiation environments.

The PIXEL-VESPA front end electronics will depart drastically from the BEETLE ASIC design. A promising prototype is the FPIX2 chip, developed for the $\mathrm{BTeV}$ pixel project. Figure 4 shows a schematic diagram of the FPIX2 pixel unit cell. It features a RC-CR preamplifier and shaper, eight comparators which provide zero suppression and form a 3-bit flash ADC and digital logic. The digital circuitry encodes the ADC information, and stores the hit information until an "output data" command is received from the end-of-column logic. Then data are transmitted to the data-output interface. The built in analog to digital conversion enhances the robustness of the system, as the information is transmitted in digital form, and the analog readout provides significant improvement in the spatial resolution [9]. In particular, a resolution between 6 and $9 \mu \mathrm{m}$, dependent upon the track angle, was achieved with a pitch of $50 \mu \mathrm{m}$. The lowest threshold is used for zero suppression, thus dramatically reducing the amount of information to be transmitted to the back end electronics. Changes to adapt this approach to the faster interaction rate and higher occupancy at LHCb are object of a planned R\&D activity.

\section{Highlights on ongoing R\&D towards the SUPER-LHCb vertex detector}

The closeness of the VELO silicon planes to the LHCb beam makes the issue of radiation hardness a primary concern. Several different approaches toward higher radiation sensitive devices are being tried in the framework of RD50 [10]. Prototype of strip and pixel sensors implemented with a variety of substrates (p-type, n-type both in float zone and magnetic Czochralski silicon substrates) have been manufactured, and exposed to different level of irradiation both from proton and neutron beams. Using the benchmark criteria of adequate collection properties at the highest levels of radiation fluence expected, silicon detectors developed on p-type substrates appear a promising option to achieve these results [11].

Recent multi-project wafer produced by collaborating institutions within the RD50 collaboration [10] have included pixel devices with a variety of geometries. In particular, pixel sensors compatible with the $\mathrm{BTeV}$ front end electronics have been designed with a joint effort between Fermilab and Syracuse University [12]. They have been characterized in the laboratory before ir- 


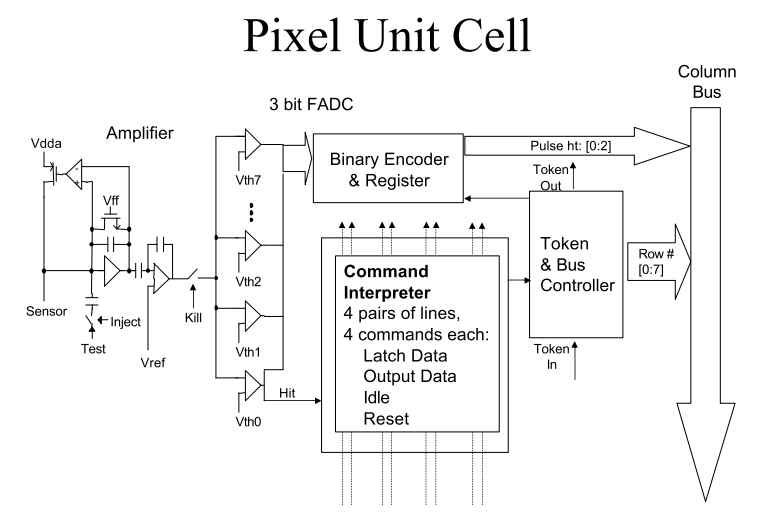

Figure 4: Schematic Diagram of the FPIX2 pixel unit cell. The analog processor is an RC-CR preamplifier and shaper followed by a discriminator to perform zero-suppression. The "flash" section encompasses additional discriminators that implement a 3-bit flash ADC. The remaining blocks represent end of cell logic and bus communication with the chip periphery.

radiation, with excellent performance. The change in behavior upon irradiation is currently under investigation.

A solution that alleviates the need for very high (500-1000 V) reverse bias applied to the silicon devices to achieve adequate charge collection would provide significantly lower demands on the high voltage distribution system and on the cooling system. These "3D detectors," constructed with Micro-Electro-Mechanical-Systems (MEMS) processing, are an innovative approach to segmented solid state detectors, where narrow holes and trenches are etched through silicon wafer and the back filled with conductive polysilicon ( $\mathrm{n}$ and $\mathrm{p}$ doped). The depletion region is achieved with lateral bias, from electrodes as close as $50 \mu \mathrm{m}$, thus requiring very modest high voltage. An additional advantage of this solution is the absence of guard ring, and the associated dead area. Almost edgeless devices can thus be implemented. This technology has been proposed by Parker and Kenney [13] in 1994, and it has undergone several years of intense development. Recent test beam data [14] have demonstrated that the technology proposed by Parker and Kenney can deliver the expected performance. The challenge is to develop a process for 3D detectors by an industrial Si device manufacturer. Several efforts are under way [14], [15], [16], and this may lead to a viable solution within a time frame compatible with the planned upgrade.

The goal to implement zero-suppressed analog readout with correct beam crossing assignment demands an active program in ASIC development, to produce a radically different front end device than the present BEETLE chip. The specifications on its architecture and data flow are closely connected with the overall data acquisition and trigger strategy. In particular, the studies of a detached vertex trigger influence the overall data flow architecture and may influence some aspects of the front end ASIC design.

New approaches to the RF shield design are critical to the material budget optimization. This is a complex task that involves investigation of different geometries, and a close interaction with CERN accelerator division scientists, especially if a coupling of the detector vacuum and of the 
beam vacuum is envisaged. Although in general silicon is not the dominant component of the material budget in the vertex detector, thinning of sensor and electronics, especially with the pixel option, are important design considerations.

\section{Conclusions}

The LHCb experiment is at the eve of a very exciting physics program that will explore several facets of flavor physics with unprecedented precision. The VELO detector is a key component to achieve its physics goals. Radiation resistance and optimal performance in a hadron trigger in a high occupancy environment are the performance criteria guiding our R\&D strategy.

\section{Acknowledgements}

This paper benefits tremendously from work presented at the "1st LHCb Collaboration upgrade workshop" [17] and from the collaborative work with T. Bowcock, P. Collins, C. Parkes, and S. Stone in drafting the VELO/VESPA upgrade R\& D plan. Finally I would like to thank S. Stone for his careful reading of this manuscript. This work was supported by the US National Science Foundation.

\section{References}

[1] [LHCb Collaboration], "Expression of interest for an LHCb upgrade," CERN-LHCC-2008-007 (2008).

[2] H. Dijkstra, In the Proceedings of 5th Flavor Physics and CP Violation Conference (FPCP 2007), Bled, Slovenia, 12-16 May 2007, pp 027 [arXiv:0708.2665 [hep-ex]].

[3] [LHCb Collaboration], "LHCb VELO TDR: Vertex locator. Technical design report,"

[4] M. Agari et al., Nucl. Instrum. Meth. A 518, 468 (2004).

[5] G. Casse, Nucl. Instr. and Meth. A 566 (2006) 26.

[6] T. Rohe, Nucl. Instr. and Meth. A460, 55 (2001).

[7] G. Casse, Nucl. Instrum. Meth. A 579, 623 (2007).

[8] E. E. Gottschalk [BTeV Collaboration], Nucl. Phys. Proc. Suppl. 156, 252 (2006).

[9] J. A. Appel et al., Nucl. Instrum. Meth. A 485, 411 (2002) [arXiv:hep-ex/0108014].

[10] The RD50 Collaboration, http://www.cern.ch/rd50.

[11] G. Casse, P.P. Allport, and A. Watson, Nucl. Instr. and Meth. A568 (2006) 46.

[12] M. Artuso, Nucl. Instrum. Meth. A 582, 835 (2007) [arXiv:physics/0701270].

[13] C. Kenney, S. Parker, J. Segal and C. Storment,

[14] A. Kok et al., Nucl. Instrum. Meth. A 560, 127 (2006).

[15] D. Pennicard et al., IEEE Trans. Nucl. Sci., V. 54, 4, 1435 (2007).

[16] A. Boscardin et al., Nucl. Instrum. Meth. A 570, 317 (2007).

[17] “ $1{ }^{\text {st }}$ LHCb Collaboration Upgrade Workshop”, January 2007, Edinburgh: http://indico.cern.ch/conferenceDisplay.py?confId=8351. 